\title{
APACHE II, SOFA and SAPS II Scoring Systems in Assessing Survival of Critically III ICU Patients.
}

\author{
Turgut Namigar, Bahadır Basak, Karacalar Serap, Mıngır Tarkan \\ Ministry of Health Okmeydani Training and Research Hospital, Department of Anesthesiology and \\ Intensive Care Unit, Istanbul, Turkey
}

\section{BACKGROUND:}

Improved mortality prediction for patients in intensive care units is a big challenge. Many severity scores have been proposed, but findings of validation studies have shown that they are not adequately calibrated. To compare the predictive ability of the most widely used scoring systems (Acute Physiology and Chronic Health Evaluation [APACHE] II, Simplified Acute Physiology Score [SAPS] II, and Sequential Organ Failure Assessment [SOFA]) for clinical outcome in patients with respiratory failure after noncardiac surgery.

\section{METHODS:}

Consecutive patients admitted to a noncardiac surgical intensive care unit (CSICU) were prospectively studied. Data on the preoperative condition, intraoperative parameters, and postoperative course were collected. The ability to predict group mortality by the SOFA score, APACHE II score, and SAPS II method was assessed using two by two decision matrices and receiver operating characteristic (ROC) curve

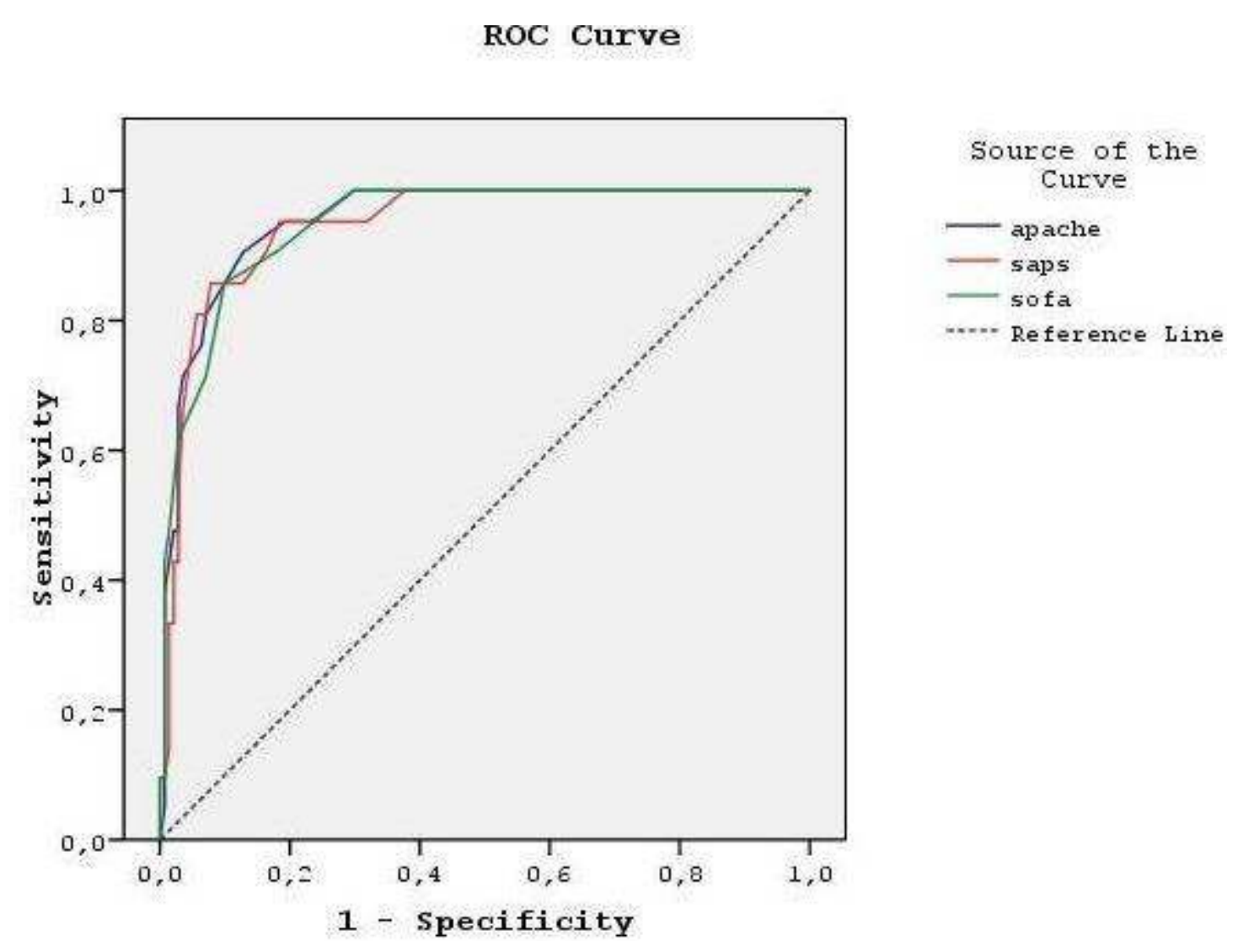
analysis.

\section{RESULTS}

-A total of 181 patients (mean age;62.7 $\pm 17.4 \mathrm{yr}$, Male;98 (\%54.1), Female;83 (\%45.9) were enrolled.

- The areas under the curve in the ROC curve (AUROC) analysis for the SOFA score, APACHE II scoring system, and SAPS II were 0.948, 0.954, and 0.946, respectively. AUROC APACHE II is only slightly higher than the other 2 AUROC incipient scoring systems.

- The cut-off value were 18.5 APACHE II ( sensitivity 90\%, specificity $87 \%$ ), 38.5 SAPS II (sensitivity 90\%, specificity $84 \%$ ), 4.5 SOFA (sensitivity $90 \%$, specificity $82 \%$ ) respectively. A strong correlation between APACHE II and SAPS II was found in this study $(p<0.01)$.

\section{CONCLUSIONS}

-The SOFA, APACHE II, and SAPS II have different capability to discriminate and estimate early in-hospital mortality of patients.

- The APACHE II score and SAPS II score are more useful in predicting mortality, and easier and simpler than the SOFA Score. 